\title{
Cognitive impairments and depression in Parkinson's disease: a follow up study
}

\author{
Sergio E Starkstein, Paula L Bolduc, Helen S Mayberg, Thomas J Preziosi, \\ Robert G Robinson
}

\begin{abstract}
The presence of depression and cognitive impairments was examined in seventy patients with Parkinson's disease (PD). Forty nine patients of this original cohort were re-examined between three and four years after the first evaluation. While both depressed and non-depressed patients showed a significant decline in cognitive function during the follow up period, intellectual decline was significantly more severe for the depressed group. Depressed patients also showed a faster rate of progression of motor signs (mainly tremor) than the non-depressed group. Patients that died during the follow up period showed significantly more cognitive impairments than patients who were alive at follow up. These findings suggest that either there may be two forms of PD: one with depression and rapid cognitive decline and one without depression and a gradual cognitive decline; or that the mechanisms of cognitive impairment in $P D$ and depression may interact to produce a more rapid evolution in cognitive impairment among PD patients with a previous depression than among patients without a previous depression.
\end{abstract}

Although depression and cognitive impairments are well known features of Parkinson's disease (PD), ${ }^{1}$ the relationship between these two factors has rarely been explored. In addition, among the investigators who have examined these two variables, some have failed to find a relationship between depression and cognitive impairment in patients with $\mathrm{PD},{ }^{2}$ while others, such as Mayeux et $a l^{3}$ have reported a significant correlation between depression and cognitive impairment (the more severe the depression the greater the cognitive impairment). Mayeux et $a^{3}$ have also suggested that patients with PD may show a "dementia of depression".

We have also recently found a significant relationship between depression and cognitive impairments in patients with PD. ${ }^{4}$ In a study of 105 patients attending an outpatient clinic, we found that severity of cognitive impairment was significantly correlated with the existence and severity of depression. ${ }^{4}$ Furthermore, the cognitive impairment associated with depression and PD was primarily shown in tasks that examined frontal lobe functions (that is, Wisconsin Card Sorting Test). In contrast, the cognitive impairments associated with PD in the absence of depression occurred in the late stages of the disease and primarily involved timed and motor tasks (for example, Trail Making Test, part A). ${ }^{5}$ Thus in our population, the intellectual impairments associated with depression in PD did not appear to represent an exacerbation of the impairments associated with PD itself, but involved different intellectual functions.

This finding then leads to questions about the nature of the relationship between cognitive impairment associated with PD and that associated with depression. For example, the cognitive impairments associated with depression and PD could be generated through entirely different neuropathological mechanisms, and the intellectual effect of the two processes could be additive. On the other hand, even if the mechanisms leading to intellectual deficits in PD patients with and without depression are different, depression and cognitive impairment in PD may interact with the progress of PD itself and produce a more rapid or severe decline in intellectual function than would otherwise occur. This suggestion is consistent with the finding that between $15 \%$ and $30 \%$ of patients with functional (that is, no known neuropathology) depression ${ }^{6}$ and between $57 \%$ and $91 \%$ of patients with dementia of depression (that is, pseudodementia) $)^{78}$ in long term follow up were no longer depressed but had developed dementia of the Alzheimer's type.

Thus this study was designed to examine the nature of the relationship between cognitive impairment associated with PD and that associated with depression. We did this by assessing the long term evolution of intellectual impairment in patients with PD and depression and comparing this with similar patients without depression.

\section{Material and methods}

From January 1983 to July 1984 a consecutive series of 70 patients with idiopathic PD who attended the neurology clinic at the Johns Hopkins Hospital (Baltimore, Maryland) were examined. Patients attending the clinic were being seen for neurological evaluation or management of their disorder at regular follow up visits.

\section{Neurological examination}

The neurological examination was carried out blind to the neuropsychological data. It consisted of a comprehensive neurological examin- 
ation and a rating scale for symptoms of PD, which quantitated the presence and severity of tremor, rigidity and akinesia (scores range from 0 to 3 for each limb) in both the left and right limbs.

\section{Psychiatric examination}

After giving informed consent, patients were given a series of standardised quantitative measures of mood and cognitive function. Examinations were administered in a private room between 10 am and $2 \mathrm{pm}$ to minimise any possible effect of diurnal mood variation. The Hamilton Rating Scale for Depression (HDS), ${ }^{9}$ a 17 item questionnaire (scores range from 0 to 52) measuring psychological and physiological symptoms of depression, was filled out by the interviewer. Patients with HDS scores of six or below were considered non-depressed, while patients with HDS scores of seven or above were considered depressed. This cut-off point was derived from a recent study ${ }^{10}$ of 105 consecutive patients with PD who were given the HDS and a standardised psychiatric evaluation (the modified PSE, ${ }^{11}{ }^{12}$ ), from which DSM-III ${ }^{13}$ diagnoses of major and dysthymic depression were generated. A cut-off score of seven on the HDS discriminated non-depressed from depressed (major or dysthymic) patients with a sensitivity and specificity of $99 \%$ and $88 \%$, respectively, mean (SD) HDS scores for major depressed patients: 15.8 (4.0), minor depressed patients: $8 \cdot 4(2 \cdot 6)$, non-depressed patients: $2 \cdot 1(0 \cdot 2)$.

The cognitive evaluation included the following tasks:

Mini-Mental State Exam (MMSE) $)^{14}$ this is an 11-item examination that has been found to be reliable in assessing a limited range of cognitive functions. Scores range from 0 to 30 , and scores of 23 or below indicate significant cognitive impairment.

Paired Associative Learning Test (PALT $)^{15}$ a series of paired words (some easy such as up-down and some difficult such as darkcrush) are read to the patients, who are later given the first word of the pair and have to respond with the second word with which it is paired.

\section{Statistical Analysis}

Statistical analysis was carried out using a two-way analysis of variance (ANOVA) with repeated measures. If a significant main effect was found, between-group comparisons were carried out with paired and non-paired Student's $t$ tests. Frequency distributions were analysed using Chi-square tests with Yates' modification for expected cell sizes below five. Correlations were calculated with Pearson's coefficients.

\section{Results}

Forty-nine $(70 \%)$ of the original cohort of 70 patients were available for the follow up examination, which was carried out between three and four years after the initial evaluation. Fourteen patients $(20 \%)$ died during the
Table 1 Demographic and neurological findings for 49 patients with and 21 without a follow up evaluation

\begin{tabular}{|c|c|c|}
\hline & $\begin{array}{l}\text { Follow up } \\
\text { mean (SD) }\end{array}$ & $\begin{array}{l}\text { Non-follow up } \\
\text { mean (SD) }\end{array}$ \\
\hline $\begin{array}{l}\text { Age (years) } \\
\text { Age at onset (years) } \\
\text { Duration of the disease (years) } \\
\text { Tremor score } \\
\text { Rigidity score } \\
\text { Akinesia score } \\
\text { Hamilton Depression score } \\
\text { Mini-Mental score } \star \star\end{array}$ & $\begin{array}{l}66.9(9.6) \\
54.8(10.4) \\
8.9(7.0) \\
1.65(0.91) \\
2.40(0.81) \\
1.65(0.73) \\
5.89(4.5) \\
28.1(2.1)\end{array}$ & $\begin{array}{l}70 \cdot 2(8 \cdot 7) \\
59 \cdot 8(10.8) \\
11.0(7.9) \\
2.00(0.95) \\
3.10(1.15) \\
1.80(0.92) \\
8.19(5.6) \\
25.6(3.5)\end{array}$ \\
\hline
\end{tabular}

$\star \star t=3.72, d f=69, p<0.001$

follow up period, four patients $(6 \%)$ were in nursing homes, away from the Baltimore area, and three patients $(4 \%)$ were lost to follow up. No significant differences in demographic variables, severity of PD symptoms, and depression scores were found between patients with and without a follow up (table 1). The only significant between-group difference was in cognitive performance (significantly worse for the group not followed up) (table 1).

The comparisons that follow include only the 49 patients who had a follow up evaluation.

\section{Demographic Findings (table 2)}

No significance between group differences were observed in demographic variables, except for education (significantly lower for depressed patients).

\section{Neurological Findings}

A two-way repeated measures ANOVA for tremor showed a significant main effect for group $(F(1,47)=7.98, p<0.01$ ) (the depressed group had a significantly more severe tremor), a significant effect for the repeated measure $(F(1,47)=5.79, p<0.05)$ (both groups showed significantly more tremor over time), and a significant interaction $(F(1,47)=$ $6.43, p<0.05)$. The depressed group showed a significantly higher increment of tremor severity during the follow up period than the non-depressed group.

A two-way repeated measures ANOVA for rigidity showed a significant main effect for group $(F(1,47)=17.46, p<0.001$ ) (the depressed group showed significantly more severe rigidity), a significant effect for the repeated measures $(F(1,47)=14.69, p$ $<0.001$ ) (both groups showed significantly more severe rigidity over time), but no significant interaction $(\mathrm{F}(1,47)=1 \cdot 25, \mathrm{p}>0.05)$.

Table 2 Demographic data for 18 depressed and 31 non-depressed patients

\begin{tabular}{lcc}
\hline & $\begin{array}{c}\text { Depressed } \\
\text { mean }(S D)\end{array}$ & $\begin{array}{l}\text { Non-depressed } \\
\text { mean }(S D)\end{array}$ \\
\hline Age (years) & $66 \cdot 0(8 \cdot 7)$ & $65 \cdot 9(10 \cdot 3)$ \\
Age at onset (years) & $54 \cdot 8(10 \cdot 5)$ & $57 \cdot 4(11 \cdot 2)$ \\
Duration of the disease (years) & $11 \cdot 1(8 \cdot 7)$ & $8 \cdot 5(6 \cdot 0)$ \\
Follow up interval (years) & $3 \cdot 8(0 \cdot 88)$ & $3 \cdot 7(0 \cdot 89)$ \\
Education (years) & $10 \cdot 9(4 \cdot 2)$ & $13 \cdot 8(3 \cdot 6)$ \\
Previous history of depression & & 16 \\
$\left({ }^{\circ}{ }_{0}\right)^{\star \star}$ & 61 & 16 \\
\hline
\end{tabular}

${ }^{*} \mathrm{t}=2.52, \mathrm{df}=48, \mathrm{p}<0.05$

$\star \star \mathrm{X}^{2}=10.5, \mathrm{df}=1, \mathrm{p}<0.01$ 
Table 3 Neurological findings

\begin{tabular}{|c|c|c|c|c|}
\hline & \multicolumn{2}{|l|}{ Depressed } & \multicolumn{2}{|l|}{ Non-Depressed } \\
\hline & Initial mean $(S D)$ & Last mean ( $S D$ ) & Initial mean (SD) & Last mean ( $S D$ ) \\
\hline $\begin{array}{l}\text { Levodopa dosage (mg/d) } \\
\text { Tremor score }{ }^{\star} \\
\text { Rigidity score } \\
\text { Akinesia score* }\end{array}$ & $\begin{array}{l}996(663) \\
1 \cdot 7(0 \cdot 9) \\
3 \cdot 1(0 \cdot 9) \\
2 \cdot 0(0 \cdot 6)\end{array}$ & $\begin{array}{l}938(558) \\
3 \cdot 3(1 \cdot 3) \\
4 \cdot 1(0 \cdot 8) \\
2 \cdot 3(0 \cdot 8)\end{array}$ & $\begin{array}{l}676(791) \\
1 \cdot 6(0.9) \\
1 \cdot 7(0.6) \\
1 \cdot 3(0 \cdot 6)\end{array}$ & $\begin{array}{l}986(775) \\
2 \cdot 5(1 \cdot 1) \\
2 \cdot 7(0.9) \\
1 \cdot 7(0 \cdot 8)\end{array}$ \\
\hline
\end{tabular}

*See text for significant findings.

A two-way repeated measures ANOVA for akinesia showed a significant main effect for group $(F(1,47)=17.36, p<0.001$ ) (the depressed group showed significantly more severe akinesia), a significant effect for the repeated measures $(F(1,47)=7 \cdot 28, p<0.01)$ (both groups showed significantly more akinesia over time), but no significant interaction $(\mathrm{F}(1,47)=0.24, \mathrm{p}>0.05)$.

A two-way repeated measures ANOVA for levodopa dosage showed no significant group $(\mathrm{F}(1,47)=0.50, \mathrm{p}>0.05)$, time $(\mathrm{F}(1,47)$ $=1.68, \mathrm{p}>0.05)$, or group by time effects $(F(1,47)=3.59, p>0.05)$. Six patients, all in the non-depressed group, were on artane at the time of the initial evaluation (dosage range: $1-10 \mathrm{mg} / \mathrm{d}$ ). Only one patient, from the nondepressed group, was put on artane $(6 \mathrm{mg} / \mathrm{d})$ during the follow up period.

\section{Psychiatric and neuropsychological findings (table 4)}

A two-way repeated measures ANOVA for HDS scores showed a significant group effect $(F(1,47)=33.11, p<0.001$. The depressed group showed significantly higher depression scores. There was no significant effect for the repeated measures $(F(1,47)=2.03, p>0.05)$, and a significant group by time interaction $(\mathrm{F}(1,47)=14.53, \mathrm{p}>0.001$. The depressed group had significantly lower scores at follow up than at first evaluation $(t=2 \cdot 7, \mathrm{df}=17$, $\mathrm{p}<0.05)$, the non-depressed group had significantly higher scores $(t=2 \cdot 07$, df $=30$, $\mathrm{p}<0.05)$ ).

Ten of the 18 patients with depression at the initial evaluation were no longer depressed at follow up, while eight of the 31 non-depressed patients at the initial evaluation were depressed at follow up. The significantly lower HDS mean score shown by the depressed group at follow up was not solely related to depression treatment. Although five of the 10 initially depressed patients who were not depressed at follow up received antidepressant medication during the follow up period, five of the remaining eight initially depressed patients who were still depressed at follow up also received antidepressant treatment. A hypothesis of increased frequency of improvement in depres- sion at follow up depending upon treatment with antidepressant medication was not statistically substantiated $\left(\chi^{2}=0 \cdot 28, \mathrm{df}=1\right.$, $\mathrm{p}>0.05$ ). It should be noted, however, that the mean (SD) daily dose of imipramine used in these patients was rather low, 28.0 (14.4) and 33.7 (19.7) $\mathrm{mg}$ for the non-depressed and still depressed groups at follow up, respectively.

On the MMSE, a two-way repeated measures ANOVA showed a significant group effect $(F(1,47)=9.82, p<0.01)$. The depressed group showed significantly lower MMSE scores, and a significant effect for the repeated measures $(F(1,47)=23.94, p<0.001)$. Both groups showed significantly lower MMSE scores over time, and a significant group by time interaction $(\mathrm{F}(1,47)=8.48, \mathrm{p}<0.01)$. MMSE scores decline was significantly greater for depressed than non-depressed patients. While first evaluation MMSE scores were not significantly different between depressed and non-depressed patients $(\mathrm{t}=1 \cdot 12, \mathrm{df}=48, \mathrm{p}>$ $0.05)$, at follow up differences became significant $(\mathrm{t}=3.38, \mathrm{df}=48, \mathrm{p}<0.01)$. Correlations between HDS and MMSE scores for the initial and follow up evaluations were not statistically significant $(r=-0.23$, $\mathrm{df}=48, \mathrm{p}>0.05$, and $\mathrm{r}=-0.01$, $\mathrm{df}=48, \mathrm{p}>0.05$, respectively). However, there was a significant correlation between HDS scores at the initial evaluation and MMSE scores at follow up $\mathrm{r}=-0 \cdot 42, \mathrm{df}=48$, $\mathrm{p}<0.01)$. That is, higher depression scores at the initial evaluation were significantly correlated with more cognitive impairment at follow up).

Since there was a significant between-group difference in years of education (table 2), differences in MMSE scores were calculated after matching depressed ( $\mathrm{n}=18$ ) and nondepressed $(n=18)$ patients for years of education ( \pm 2 years), mean $(S D)$ years $11 \cdot 8(3 \cdot 1)$ and $12.3(2.9)$, respectively. A two-way repeated measures ANOVA showed similar results, since there was still a significant group effect $(\mathrm{F}(1,34)=9.01, \mathrm{p}<0.01)$, a significant effect for the repeated measures $(F(1,34)=16.98$, $\mathrm{p}<0.01)$, and a significant interaction $(\mathrm{F}(1,34)$ $=4.41, \mathrm{p}<0.05)$, mean (SD) MMSE scores (first evaluation) for depressed and non-

Table 4 Psychiatric and neuropsychological findings

\begin{tabular}{|c|c|c|c|c|}
\hline & \multicolumn{2}{|l|}{ Depressed } & \multicolumn{2}{|l|}{ Non-Depressed } \\
\hline & Initial mean $(S D)$ & Last mean $(S D)$ & Initial mean $(S D)$ & Last mean ( $S D$ ) \\
\hline $\begin{array}{l}\text { Mini-Mental (score) }{ }^{\star} \\
\text { PALT (score) } \\
\text { Hamilton Depression Scale }\end{array}$ & $\begin{array}{l}27 \cdot 7(2 \cdot 3) \\
11 \cdot 2(2 \cdot 7) \\
10 \cdot 6(3 \cdot 5)\end{array}$ & $\begin{array}{r}23 \cdot 0(6 \cdot 0) \\
8 \cdot 4(1 \cdot 9) \\
7 \cdot 1(4 \cdot 7)\end{array}$ & $\begin{array}{r}28.5(2.0) \\
11.8(3.4) \\
3.1(1.9)\end{array}$ & $\begin{array}{r}27 \cdot 3(2 \cdot 7) \\
11 \cdot 3(3 \cdot 7) \\
4 \cdot 7(4 \cdot 5)\end{array}$ \\
\hline
\end{tabular}

$\star$ See text for significant findings. 
depressed patients: $27 \cdot 8(2 \cdot 3)$ and $29 \cdot 1(0 \cdot 9)$, respectively (paired $t=2 \cdot 44, \mathrm{df}=17$, $\mathrm{p}<0.05$ ), and (follow up evaluation) $23.3(6.0)$ and $27.5(2.6)$ respectively (paired $t=2.64$, $\mathrm{df}=17, \mathrm{p}<0.05)$ ).

Since depressed and non-depressed patients also differed, though not significantly, in duration of the disease, MMSE score differences were calculated for depressed $(n=17)$ and non-depressed ( $\mathrm{n}=17$ ) pairs matched for duration of the disease ( \pm 2 years), mean years (SD) $9.41(4.7)$ and $9 \cdot 70$ (4.75), respectively. One patient from the depressed group (with a duration of the disease of 35 years) could not be matched with a non-depressed patient. Again, a two-way ANOVA with repeated measures showed a significant group effect $(F(1,32)=4.53, p<0.05)$, a significant effect for the repeated measures $(F(1,32)=11 \cdot 56, p$ $<0.01)$, and a significant interaction $(F(1,32)$ $=6.40, p<0.05)$ (MMSE scores for the first evaluation were $28.0(2.23)$ and $28.2(2.51)$, paired $t=0.36, \mathrm{df}=16, \mathrm{p}>0.05$, for depressed and non-depressed patients respectively), while at follow up, scores were 23.5 $(6 \cdot 20)$ and $27.6(1.62)$, respectively (paired $t$ $=2.32, \mathrm{df}=16, \mathrm{p}<0.05))$.

Since depressed and non-depressed patients differed in the severity of tremor, rigidity, and akinesia, MMSE scores differences were calculated for depressed $(n=15)$ and nondepressed $(n=15)$ pairs matched ( \pm 2 points) for the global score of motor symptoms (scores for tremor, rigidity, and akinesia were added), mean (SD) global scores were $6.80(2.9)$ for depressed, and 6.33 (3.1) for non-depressed patients. Three patients from the depressed group (with global scores of 8,10 , and 11) could not be matched with non-depressed patients. A two-way ANOVA with repeated measures showed a significant group effect $(F(1,28)=$ $11.8, p<0.01)$, a significant effect for the repeated measures $(F(1,28)=12.0, p<0.01)$, and a significant interaction $(\mathrm{F}(1,28)=5.64$, $\mathrm{p}<0.05$ ), mean (SD) MMSE scores (first evaluation) for depressed and non-depressed patients were $27 \cdot 7(2 \cdot 2)$ and $29.0(0 \cdot 8)$, respectively (paired $t=2 \cdot 0, \mathrm{df}=14, \mathrm{p}>0.05$ ), and (follow up evaluation), 22.7 (6.1) and $28 \cdot 1$ (2.7), respectively (paired $t=2 \cdot 88, \mathrm{df}=14$, $\mathrm{p}<0.05)$.

Finally, the possibility that antidepressant medication may have influenced MMSE results was also examined. While the initial MMSE scores for treated and non-treated depressed patients were not significantly different, $27.5(2.5)$ and $28.2(2.05)$, respectively, follow up scores were lower (more impaired) for the non-treated group, $21 \cdot 8(7 \cdot 5)$, $23 \%$ decrement, than for the treated group, $24 \cdot 6(4 \cdot 7), 11 \%$ decrement. Thus antidepressant medication may have diminished the rate of progression of cognitive impairment. In fact, the two patients that received the highest dosage of antidepressants (imipramine $50 \mathrm{mg}$ / d) and were not depressed at follow up, showed no change in MMSE scores over time. Both scored 30 points at the initial and follow up evaluations.

On the PALT, there was a significant effect for the repeated measures $(F(1,47)=5 \cdot 58, p<$ $0.05)$. While between-group differences were not significant at the initial evaluation $(t=$ $0.89, \mathrm{df}=48, \mathrm{p}>0.05)$, there was a trend for a significant interaction $(\mathrm{F}(1,47)=2 \cdot 67, \mathrm{p}=$ 0.09 ), and the depressed group showed significantly lower scores at follow up $(t=2 \cdot 34$, df $=48, \mathrm{p}<0.05)$.

To determine whether the cognitive decline was faster among those depressed patients who were still depressed at follow up, we divided the depressed group into those who were not depressed at follow up (HDS score $<7)(\mathbf{n}=$ 10), and those who were still depressed (HDS score equal or $>7)(n=8)$. Mean (SD) HDS scores for the recovered group were $9 \cdot 7(2 \cdot 1)$ and $3.7(2.0)$, and for the non-recovered group $11.8(4.6)$ and $11.2(3.3)$ (initial and follow up evaluations, respectively). Age at follow up, mean years (SD) for the recovered group was $65.9(8.6)$, and for the non-recovered group was $72.0(8.3)(t=1.50, \mathrm{df}=16, \mathrm{p}>0.05)$. MMSE scores for the recovered group were $27.9(2.3)$ and $22.5(6.4)$, and for the nonrecovered group $27.8(2.3)$ and $24.5(5.8)$ (initial and follow up evaluations, respectively). A two-way ANOVA with repeated measures showed a significant effect for the repeated measures $(F=10$, df $=1,16, p<0.01$ ) (both groups showed a significant decline in MMSE during the follow up period), but no significant interaction $(\mathrm{F}=0.62, \mathrm{df}=1,16$, $p>0.05$ ) (the decline was similar for both, recovered and non-recovered groups).

When patients that were non-depressed at the initial evaluation were divided, based on the follow up evaluation, into depressed $(n=8)$ and non-depressed $(n=23)$ groups, a two-way ANOVA with repeated measures for MMSE scores showed no significant group $(F(1,29)=$ $2 \cdot 21, p>0.05)$ or interaction effects $(F(1,29)$ $=0.05, p>0.05)$, but a significant time effect $(F(1,29)=4.30, p<0.05)$, MMSE scores mean (SD) for the depressed group: initial 29.3 $(0 \cdot 8)$, follow up $28.2(1.2)$; for the non depressed: initial $28 \cdot 1(2 \cdot 2)$, follow up $26 \cdot 9(2 \cdot 9)$.

When, based on the follow up evaluation, patients were divided into depressed $(n=16)$ and non-depressed ( $n=33$ ) groups, no significant differences in MMSE scores were observed. MMSE scores, mean (SD): depressed group: $26.6(4.3)$, non-depressed: 26.4 $(4 \cdot 5) ; \mathrm{t}=0 \cdot 25, \mathrm{df}=47, \mathrm{p}>0.05)$.

Comparison between patients who survived and patients who died during the follow up period: Fourteen of the original cohort of 70 patients died during the follow up period. When they were compared with the 53 patients who were alive at follow up, significant differences emerged (table 5). Deceased patients were significantly older $(t=3.73, \mathrm{df}=66, \mathrm{p}<0.01)$, and had significantly worse MMSE and PALT scores $(\mathrm{t}=3.69$, df $=66, \mathrm{p}<0.001$, and $\mathrm{t}=2.82, \mathrm{df}=66, \mathrm{p}<0.01$, respectively).

When deceased $(n=14)$ and non-deceased patients $(n=14)$ were matched for age $( \pm 2$ years), 74.4 (5.97) and 73.3 (5.45), respectively, between-group differences on cognitive tasks 
Table 5 Demographic, neurological, and neuropsychiatric differences between 14 deceased and 53 non-deceased patients

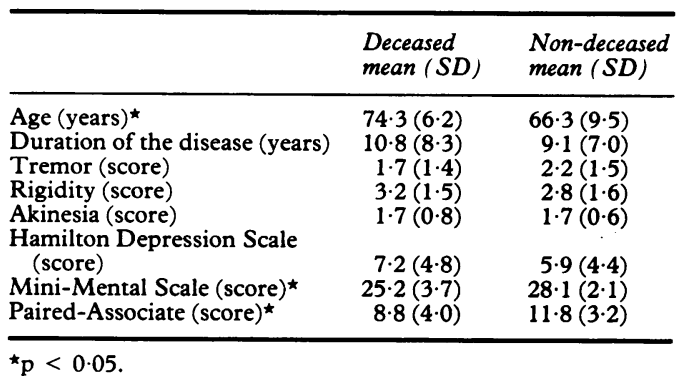

still remained significant $(M M S E$ deceased: $25 \cdot 2(3 \cdot 75)$, non-deceased: $28 \cdot 2(1 \cdot 67)$, paired $t$ $=3.00, \mathrm{df}=13, \mathrm{p}<0.01 ; P A L T$ deceased: $8 \cdot 17$ (4.35), non-deceased: $11.85(3.51)$, paired $t=2 \cdot 18, \mathrm{df}=13, \mathrm{p}<0.05)$. On the other hand, duration of the disease was not significantly different (deceased: $10 \cdot 7(8.4)$ years, non-deceased: $9.6(9.2)$ years, paired $t=0.46$, $\mathrm{df}=13, \mathrm{p}>0.05)$. No significant betweengroup differences were observed in the severity of depression (HDS deceased: $7 \cdot 2$ (4.8) nondeceased: $6.5(4.4)$, tremor (deceased: 1.71 (1.48), non-deceased: $2 \cdot 21(1.52)$, paired $t=$ $0 \cdot 86, \mathrm{p}>0.05$, rigidity (deceased: $3.21(1.57)$, non-deceased: $2.85(1.61)$, paired $t=0.56, \mathrm{p}>$ $0.05)$, and akinesia (deceased: $1.78(0.8)$, nondeceased: $1.71(0.61)$, paired $t=0.80, \mathrm{p}>$ 0.05).

\section{Discussion}

Our study found that patients with PD showed a significant decline in cognitive function during a three to four year follow up period. In addition, patients with PD who were depressed at the time of their initial evaluation showed a significantly greater degree of cognitive decline than those who were not depressed. The presence of depression was associated with a subsequent loss of intellectual function even when the depression was no longer present. These greater impairments among the group with PD and depression were evident on a scale of global cognitive impairment as well as on a memory task, and suggest that the presence of a depressive episode may accelerate the progression of cognitive decline in patients with $P D$.

Before further discussion, one limitation of this study should be addressed. Patients were divided into depressed and non-depressed groups based on a cut-off score of the HDS, and standardised diagnostic criteria for depression were not used. The cut-off score used in our study, however, proved to have high sensitivity and specificity for (major and dysthymic) depression in patients with PD (see Material and methods).

Similar to recent findings by Huber et al, ${ }^{2}$ depressed patients in this study showed significantly more rigidity and akinesia than nondepressed patients. A new finding, however, shows that depressed patients developed significantly more severe tremor over time than the non-depressed group. While no significant group by time interaction was observed for either rigidity or akinesia, significant differences at the initial evaluation may have produced a "ceiling-effect" for depressed patients' scores. In any event, the finding that depressed patients with PD showed more severe and/or a faster progression of PD symptoms deserves further study.

How might the finding of significantly lower MMSE scores at follow up for the depressed group compared with the non-depressed group be construed? In the introduction, we suggested that cognitive impairments associated with depression in PD and cognitive impairments associated with PD alone may either be two independent processes, or may interact in some way. The results from our study do not support the hypothesis that intellectual impairment associated with depression and cognitive impairment associated with PD are independent processes. The fact that patients with initial depression showed a more rapid decline in intellectual function than patients with PD alone (that is, there was a significant interaction between depression and intellectual function on the two-way ANOVA) indicates that the cognitive effects of depression in PD are not simply additive to those associated with $\mathrm{PD}$ alone. It is possible that there are two different forms of $P D$, one with depression and rapid cognitive decline and a second without depression and a more gradual cognitive deterioration. It is also possible, however, that the mechanism of cognitive decline associated with PD may have interacted with the mechanism of cognitive impairment associated with depression to produce a greater decline in cognition that would be attributable to either depression or PD alone.

If one assumes that there is an interaction between cognitive impairment in PD and cognitive impairment in depression with $\mathrm{PD}$, the question then becomes, how might this interaction occur? Some studies have reported that the dementia in PD correlates with the presence of Alzheimer's disease type pathological changes (plaques and tangles), ${ }^{16}$ or the severity of depletion of cholinergic neurons in the basal forebrain. ${ }^{17}$ On the other hand, Mayeux et $a l^{18} 19$ showed that patients with PD and depression had significantly lower 5-HIAA (a metabolite of serotonin) levels in the CSF than patients with PD without depression. In addition, Torack and Morris ${ }^{20}$ have recently found a marked loss of pigmented neurons in the ventral tegmental area (VTA) in a small group of patients with PD, dementia and depression. The VTA contains cell bodies of dopaminergic neurons that provide most of the dopaminergic innervation of the prefrontal cortex, limbic areas (hippocampus and amygdala) and subcortical structures with limbic and notor connections (nucleus accumbens). ${ }^{21}$

Patients with functional depression (that is, depression with no known brain lesion) have plasma hypercortisolaemia, which may result in brain atrophy. ${ }^{22}$ Rubinow et $a l^{22} 23$ showed a significant correlation between hypercortisolaemia, brain atrophy and cognitive impairment in patients with functional depression. 
Furthermore, Sroka et $a l^{24}$ reported that patients with PD and an organic brain syndrome have significant cortical atrophy, and depressed patients with PD may show nonsuppression on the dexamethasone suppression test. ${ }^{25}$

Thus, through one or a combination of several neuropathological processes (that is, serotonergic neuronal dysfunction, mesolimbic dopaminergic dysfunction, or hypercortisolaemia with secondary generalised brain atrophy), the cognitive impairment associated with depression may occur. The mechanism by which these neuropathological processes are expressed as cognitive impairment may be influenced by the nigrostriatal pathway degeneration that is inherent to $P D$, and in some patients, by Alzheimer type degenerative changes. The interaction of these processes over time could result in a greater degree of cognitive impairment than would otherwise result from the simple addition of these two cognitive impairments.

The finding that patients with PD and depression have a worse prognosis for intellectual deterioration over time than non-depressed patients has two major implications. First, it suggests that adequate treatment of depression may be of potential help in preventing or delaying the progression of cognitive deficits. This suggestion is supported by our finding that patients treated for depression had only an $11^{\circ}{ }_{0}$ decrease in cognitive scores compared to a $23^{\circ}{ }_{0}$ decrement in cognitive scores for nontreated depressed patients. Moreover, the two patients with the highest dose of antidepressants showed no cognitive decline. Second, the group of patients that died during the follow up period were significantly more cognitively impaired than patients who were alive at follow up, and this finding held true even when differences in age and duration of illness were controlled. While this significant association does not allow a statement of causality, it may be speculated there are two different forms of $P D$, one with depression followed by a rapid cognitive decline and a shorter life expectancy, and a second one in which the cognitive decline is more gradual and the life expectancy longer.

In conclusion, intellectual deterioration in patients with PD appears to be more rapid when there has been coexisting depression. The greater degree of intellectual deterioration also appears to be associated with a shorter life expectancy in patients with PD. Although we cannot determine whether these findings indicate that the cognitive impairment results from an interaction between depression and PD or indicates that there is more than one type of PD, these findings suggest important variables for further investigation.

This work was supported in part by the following National Institutes of Health grants: Research Scientist
Development Award MH-00163 (RGR), NS-15178, NS-18622, NS-15080, and MH-40355, and in part by a grant from the University of Buenos Aires (SES) and a National Alliance for Research on Schizophrenia and Depression (NARSAD) Fellowship Extension Award (SES).

1 Mayeux R, Stern Y, Williams JBW, Sano M, Cote L. Depression and Parkinson's disease. Adv Neurol 1986; 45:451-5.

2 Huber SJ, Paulson GW, Shuttleworth EC. Relationship of motor symptoms, intellectual impairment, and depression in Parkinson's disease. J Neurol Neurosurg Psychiatry 1988;51:855-8.

3 Mayeux R, Stern Y, Rosen J, Leventhal J. Depression, intellectual impairment and Parkinson's disease. Neurology 1981;31:645-50.

4 Starkstein SE, Preziosi TJ, Berthier ML, Bolduc PL, Mayberg HS, Robinson RG. Depression and cognitive impairments in Parkinson's disease. Brain 1989; 112:1141-53.

5 Starkstein SE, Preziosi TR, Robinson RG. Cognitive impairment in the different stages of Parkinson's disease. Journal of Neuropsychiatry and Clinical Neurosciences Journal of $N$.

6 Gustafson L. Psychiatric symptoms in dementia with onset in the presenile period. Acta Psychiatr Scand 1975;257: 9-35.

7 Reding M, Haycox J, Blass J. Depression in patients referred to a dementia clinic: a three-year prospective study. Arch Neurol 1985;42:894-6.

$8 \mathrm{Kral}$ VA. The relationship between senile dementia (Alzheimer type) and depression. Can J Psychiatry 1983; 28:304-6.

9 Hamilton MA. A rating scale for depression. J Neurol Neurosurg Psychiatry 1960;23:56-62.

10 Starkstein SE, Preziosi TJ, Robinson RG. Depression in Parkinson's disease. J Nerv Ment Dis 1990;178:27-31.

11 Robinson RG, Kubos KL, Starr LB, Rao K, Price TR. Mood changes in stroke patients: relationship to lesion Mood changes in stroke patients: relations

12 Wing JK, Cooper JK, Sartorius N. Measurements and Classification of Psychiatric Symptoms. New York: Cambridge University Press, 1974.

13 American Psychiatric Association. Diagnostic and statistical manual of mental disorders (DSM-III). Third ed. Washington DC, American Psychiatric Association, 1980

14 Folstein MF, Folstein SE, McHugh PR. "Mini-mental state": a practical method for grading the cognitive state of patients for the clinician. J Psychiatr Res 1975;12:189-98.

15 Wechsler D. A standardized memory scale for clinical use. $J$ Psychol 1945;18:87-95.

16 Boller F, Mizutani T, Roessmann U, Gambetti P. Parkinson's disease, dementia and Alzheimer disease: clinico-pathological correlations. Ann Neurol 1980;1: 329-55.

17 Perry EK, Curtis M, Dick DJ, Candy JM, Atack JR, Bloxham CA, Blessed G, Fairbairn A, Tomlinson BE, Perry RH. Cholinergic correlates of cognitive impairmen in Parkinson's disease: comparisons with Alzheimer's disease. J Neurol Neurosurg Psychiatry 1985;48:413-21.

18 Mayeux R, Stern Y, Williams JBW, Cote L, Frantz A Dyrenfurth I. Clinical and biochemical features of depression in Parkinson's disease. Am J Psychiatry 1986; 143:756-9.

19 Mayeux R, Stern Y, Sano M, Williams JBW, Cote LJ. The relationship of serotonin to depression in Parkinson's disease. Movement Disorders 1988;3:237-44.

20 Torack RM, Morris JC. The association of ventral tegmental area histopathology with adult dementia. Arch Neurol

21 Simon $\mathrm{H}$, Le Moal M, Calas A. Efferents and afferents of the ventral-tegmental-A10 region studied after local injection of $\left[{ }^{3} \mathrm{H}\right]$ leucine and horseradish peroxidase. Brain Res 1979;1 17: $17-40$.

22 Kellner CH, Rubinow DR, Gold PW, Post RM. Relationship of cortisol hypersecretion to brain CT scan alterations in depressed patients. Psychiatry Res 1983;8:191-7.

23 Rubinow DR, Post RM, Savard R, Gold PW. Cortisol hypersecretion and cognitive impairment in depression. Arch Gen Psychiatry 1984;41:279-83.

24 Sroka H, Elizan TS, Yahr MD, Burger A, Mendoza MR. Organic mental syndrome and confusional states in Parkinson's disease: relationship to computerized tomographic signs of cerebral atrophy. Arch Neurol 1981;38: 339-42.

25 Frochtengarten ML, Villares JCB, Maluf E, Carlini EA Depressive symptoms and the dexamethasone suppression test in Parkinsonian patients. Biol Psychiatry 1987;
sion 22:386-9. 